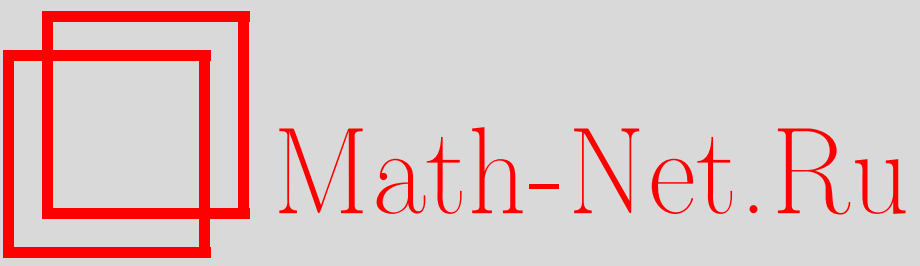

А. Д. Суворов, Группа вращений ребер композиции плоских деревьев и сплетение групп, УМH, 1999, том 54, выпуск 1, 267-268

DOI: https://doi.org/10.4213/rm131

Использование Общероссийского математического портала Math-Net.Ru подразумевает, что вы прочитали и согласны с пользовательским соглашением

http://www.mathnet.ru/rus/agreement

Параметры загрузки:

IP: 54.162 .85 .209

26 апреля 2023 г., 17:58:41 


\title{
ГРУППА ВРАЩЕНИЙ РЕБЕР КОМПОЗИЦИИ ПЛОСКИХ ДЕРЕВЬЕВ И СПЛЕТЕНИЕ ГРУПП
}

\author{
А. Д. Суворов
}

1. Плоские двукрашенные деревья. В этой работе будут рассматриваться плоские двукрашенные деревья. Как обычно, дерево - связный граф̆ без циклов. Вершины разбиты на два множества (черных и белых вершин), так что соседние вершины - разного цвета. Плоская структура означает, что в каждой вершине задан циклический порядок ребер. Будем использовать обозначения $E(T)$ и $V_{\bullet}(T)\left(V_{\circ}(T)\right)$ для множества ребер дерева $T$ и множеста черных (белых) вершин этого дерева.

Группой вращений ребер $E R(T)$ плоского двукрашенного дерева $T$ называется группа перестановок ребер дерева $T$, порожденная элементами $a_{\bullet}, a_{\circ}-$ вращениями ребер вокруг черных и белых вершин дерева [1].

Для того чтобы задать плоское двукрашенное дерево $T$, достаточно указать множество ребер $E(T)$ и перестановки $a_{\bullet}, a_{\circ}$ (вершины соответствуют независимым циклам перестановок $a_{\bullet}, a_{\circ}$, очевидным образом определяется отношение инцидентности).

Легко определить плоское двукрашенное дерево "ежик"- $H_{n}=\left(E=\{1, \ldots, n\}, a_{\bullet}=\mathrm{id}\right.$, $\left.a_{\circ}=(1,2, \ldots, n)\right)$ и "цепочку" $-C_{n}=\left(E=\{1, \ldots, n\}, a_{\bullet}=(1,2) \ldots(n-2, n-1), a_{\circ}=\right.$ $(2,3) \ldots(n-1, n))$, если число ребер $n$ нечетно, и $C_{n}=\left(E=\{1, \ldots, n\}, a_{\bullet}=(1,2) \ldots(n-1, n)\right.$, $\left.a_{\circ}=(2,3) \ldots(n-2, n-1)\right)$, если $n$ четно.

Группа вращений ребер ежика $H_{n}$ - циклическая группа $\mathbb{Z}_{n}$, группа вращений ребер цепочки $C_{n}$ - диэдральная группа $D_{n}(n \geqslant 3)$.

Морфизмом абстрактных плоских двукрашенных деревьев $T=\left(E, a_{\bullet}, a_{\circ}\right)$ и $T^{\prime}=\left(E^{\prime}, a_{\bullet}^{\prime}, a_{\circ}^{\prime}\right)$ называется отображение $\varphi: E \rightarrow E^{\prime}$, удовлетворяющее условиям $\varphi \circ a_{\bullet}(e)=a_{\bullet}^{\prime} \circ \varphi(e), \varphi \circ$ $a_{\circ}(e)=a_{\circ}^{\prime} \circ \varphi(e), \forall e \in E$.

Группа вращений ребер "наугад взятого" дерева $T$ с $n$ ребрами $-S_{n}$ или $A_{n}$ в зависимости от четности порождающих перестановок $a \bullet, a_{\circ}$. Точнее, имеет место один из следующих трех случаев.

i) Общий случай - группа $E R(T) \simeq S_{n}$ или $E R(T) \simeq A_{n}$.

ii) Дерево $T$ является ежиком или цепочкой - группа $E R(T) \simeq \mathbb{Z}_{n}$ или $E R(T) \simeq D_{n}$.

iii) Группа $E R(T)$ примитивна и отлична от $S_{n}, A_{n}, \mathbb{Z}_{n}, D_{n}$. Тогда дерево $T$ называется $u c-$ ключительным. деревом. Получен полный список исключительных деревьев (см. [2], [3]).

iv) Группа $E R(T)$ импримитивна. Этот случай имеет место тогда и только тогда, когда существует морфизм $\varphi: T \rightarrow T^{\prime}, 1<\# E\left(T^{\prime}\right)<\# E(T)$. Дерево $T$ называется в этом случае приводимым.

В частности, дерево $T$ является приводимым, если оно является результатом нетривиальной композиции деревьев: $T=T_{1} \circ T_{2} ; \# E\left(T_{1}\right)$, \# $E\left(T_{2}\right)>1$.

2. Группа врашений ребер композиции плоских двукрашенных деревьев. Следуя работе [4], определим композицию плоских двукрашенных деревьев с двумя отмеченными вершинами.

Пусть $T=\left(E, a_{\bullet}, a_{\circ}, v_{\square}, v_{\triangle}\right)$ - плоское двукрашенное дерево с отмеченными вершинами $v_{\square}, v_{\triangle} \in V(T)$. Однозначно определен путь $\sigma$ в дереве $T$, соединяющий вершины $v_{\square}, v_{\triangle}$. В пути $\sigma$ существует единственное ребро $e_{\square}$, инцидентное $v_{\square}$, и единственное ребро $e_{\triangle}$, инцидентное $v \triangle$

Опишем абстрактное плоское двукрашенное дерево с двумя отмеченными вершинами $T_{3}=$ $\left(E_{3}, a_{\bullet 3}, a_{\circ 3}, v_{\square 3}, v_{\triangle 3}\right)$ - композицию абстрактных плоских двукрашенных деревьев с двумя отмеченными вершинами $T_{1}=\left(E_{1}, a_{\bullet 1}, a_{\circ 1}, v_{\square 1}, v_{\triangle 1}\right)$ и $T_{2}=\left(E_{2}, a_{\bullet 2}, a_{\circ 2}, v_{\square 2}, v_{\triangle 2}\right)$.

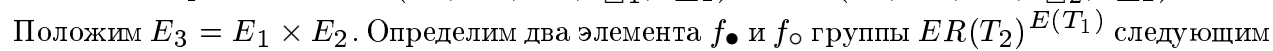
образом:

$$
f_{\bullet}(e)=\left\{\begin{array}{l}
\operatorname{id}_{2}, \text { если } e \neq e_{\square}, e_{\triangle}, \\
a_{\bullet 2}, \text { если } e=e_{\square} \text { и } v_{\square} \in V_{\bullet}, \\
a_{\circ 2}, \text { если } e=e_{\triangle} \text { и } v_{\triangle} \in V_{\bullet},
\end{array} \quad f_{\circ}(e)=\left\{\begin{array}{l}
\operatorname{id}_{2}, \quad \text { если } e \neq e_{\square}, e_{\triangle}, \\
a_{\bullet 2}, \text { если } e=e_{\square} \text { и } v_{\square} \in V_{\circ}, \\
a_{\circ 2}, \text { если } e=e_{\triangle} \text { и } v_{\triangle} \in V_{\circ} .
\end{array}\right.\right.
$$

Положим $a_{\bullet 3}\left(e_{1}, e_{2}\right)=\left(a_{\bullet 1}\left(e_{1}\right),\left(f \bullet\left(e_{1}\right)\right)\left(e_{2}\right)\right) ; \quad a_{\circ 3}\left(e_{1}, e_{2}\right)=\left(a_{\circ 1}\left(e_{1}\right),\left(f_{\circ}\left(e_{1}\right)\right)\left(e_{2}\right)\right) ;$ $a_{\bullet 3}, a_{\circ 3} \in E R\left(T_{1}\right)$ 乙 $E R\left(T_{2}\right)$. Для завершения конструкции следует определить отмеченные 
вершины дерева $T_{3}=T_{1} \circ T_{2}$ :

$$
\begin{gathered}
v_{\square 3}= \begin{cases}\text { орбита ребра }\left(e_{\square 1}, e_{\square 2}\right) \text { в } a_{\bullet 3}, & \text { если } v_{\square 2} \in V_{\bullet 2}, \\
\text { орбита ребра }\left(e_{\triangle 1}, e_{\square 2}\right) \text { в } a_{\circ 3}, & \text { если } v_{\square 2} \in V_{\circ 2} .\end{cases} \\
v_{\triangle 3}= \begin{cases}\text { орбита ребра }\left(e_{\square 1}, e_{\triangle 2}\right) \text { в } a_{\bullet 3}, & \text { если } v_{\triangle 2} \in V_{\bullet 2}, \\
\text { орбита ребра }\left(e_{\triangle 1}, e_{\triangle 2}\right) \text { в } a_{\circ 3}, & \text { если } v_{\triangle 2} \in V_{\circ 2} .\end{cases}
\end{gathered}
$$

Группа вращений ребер композиции плоских деревьев включается в следующую диаграмму:

$$
\begin{aligned}
& 1 \\
& \text { (i) } \quad 1 \longrightarrow \quad \begin{array}{c}
K \\
\downarrow i_{1}
\end{array} \quad \stackrel{\alpha^{\prime}}{\longrightarrow} \quad \begin{array}{c}
E R\left(T_{1} \circ T_{2}\right) \\
\downarrow i_{2}
\end{array} \quad \stackrel{\pi^{\prime}}{\longrightarrow} E R\left(T_{1}\right) \longrightarrow 1
\end{aligned}
$$

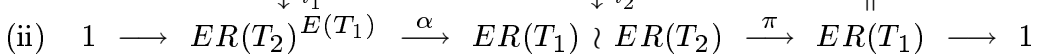

$$
\begin{aligned}
& \alpha(f)=(1, f) ; \quad \pi(g, f)=g .
\end{aligned}
$$

Вообще говоря, $E R\left(T_{1} \circ T_{2}\right)$ не является нормальной подгруппой в $E R\left(T_{1}\right)$ 乙 $E R\left(T_{2}\right)$.

Обозначим $k_{c}:=\left[E R\left(T_{1}\right) \prec E R\left(T_{2}\right): E R\left(T_{1} \circ T_{2}\right)\right]=\left[E R\left(T_{2}\right)^{E\left(T_{1}\right)}: K\right]$. Ниже приведены результаты о возможных значениях этого индекса.

Наиболее удобным для исследования является случай $T_{2}=H_{p}$. Тогда расшшрения (i) и (ii) абелевы, следовательно определено действие группы $E R\left(T_{1}\right)$ сопряжениями в $K$ и $E R\left(T_{2}\right) E\left(T_{1}\right)$, которое будет обозначаться $L: E R\left(T_{1}\right) \rightarrow \operatorname{Aut}\left(E R\left(T_{2}\right)^{E\left(T_{1}\right)}\right)$ (действие является линейным). Изучение аффинных продолжений этого действия позволило получить следующие результаты.

В оставшихся утверждениях будем придерживаться следуюших соглашений: дерево $T_{2}=H_{p}$, центральная вершина белого цвета; отмеченная вершина $v \triangle$ дерева $T_{1}$ - черного цвета.

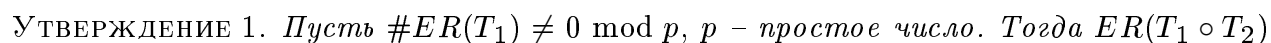
является собственной подгруппой $E R\left(T_{1}\right)$ 乙 $E R\left(T_{2}\right)$ тогда и только тогда, когда на некотором нетривиалном факторпредставлении $S$ представления $E R\left(T_{2}\right)^{E\left(T_{1}\right)}$ группь $E R\left(T_{1}\right)$ имеет решение следующая система линейньх уравнений:

$$
\left\{\begin{array}{l}
L_{a_{\bullet 1}} x-x+L_{a_{\bullet}} f_{\bullet}=0 \\
L_{a_{\circ 1}} x-x=0
\end{array}\right.
$$

Условие разрешимости системь является достаточным для нетривиальности индекса $k_{c}$ при любом порядке группы $E R\left(T_{1}\right)$ (см. утвержсдение 3$)$.

УтвеРЖДЕнИЕ 2. Пусть $E R\left(T_{1}\right)=S_{n}$ или $A_{n}$. Тогда при подходящем выборе вершинь $v_{\triangle}$ могут иметь место следующие значения индекса: $k_{c}=2$, если $p=2$, $E R\left(T_{1}\right)=S_{n}$, образующая $a_{\bullet 1}$ нечетна, $a_{\circ 1}$ четна; $k_{c}=p^{n-1}$ иначе.

УТВЕРЖДЕнИЕ 3. Пусть все чернье вершины дерева $T_{1}$, кроме $v_{\triangle}$, имеют валентности, кратные $p$. Тогда для композиции $T_{3}=T_{1} \circ T_{2}$ имеем $k_{c}=p^{n-1}$.

В случае выполнения условий утверждения 3 существует морфизм $\varphi: T_{1} \circ T_{2} \rightarrow H_{p}$ дерева $T_{1} \circ T_{2}$ на ежик с $p$ ребрами (вообще говоря, $T_{1} \circ T_{2}$ не представляется в виде композиции $H_{p} \circ T$ ежика $H_{p}$ с каким-либо деревом $T$ ). Описанный в утверждении 3 класс деревьев соответствует неоднозначно разложимым полиномам (см. [5]).

\section{СПИСОК ЛИТЕРАТУРЫ}

[1] Адрианов Н. М., Кочетков Ю. Ю., Суворов А. Д., Шабат Г. Б. // Фунд. прикл. матем. 1995. Т. 1. № 2. С. 377-384. [2] Адрианов Н. М., Кочетков Ю. Ю., Суворов А. Д. // Фунд. прикл. матем. 1997. V. 3. № 4. Р. 1091-1098. [3] Адрианов Н. М. // Фунд. прикл. матем. 1997. Т. 3. № 4. C. 1075-1089. [4] Adrianov N. M., Zvonkin A. K. // Acta Appl. Math. 1998. № 1-3. Р. 239-245. [5] Суворов А. Д. О приводимых деревьях // Kurosh Algebraic Conference'98, Москва, 1998. С. 214-215. 